\title{
中国石松类和蕨类植物的红色名录评估
}

\author{
董任勇 $1^{*}$ 左政裕 ${ }^{1,2}$ 严岳酒 ${ }^{3}$ 向建英 ${ }^{4}$ \\ 1 (中国科学院华南植物园植物资源保护与可持续利用重点实验室，广州 510650) \\ 2 (中国科学院大学, 北京 100049) \\ 3 (上海辰山植物园, 中国科学院上海辰山植物科学研究中心, 上海 201602) \\ 4 (西南林业大学云南生物多样性研究院, 昆明 650224)
}

\begin{abstract}
摘要: 为了解中国现存石松类和蕨类植物的灭绝风险, 我们按照IUCN Red List Categories and Criteria (Version 3.1)首次开展了国家水平上的红色名录评估。三人评估小组通过查阅文献、标本以及向其他30位中国蕨类同行征 询物种信息等途径, 历经 16 个月的时间完成了此次评估。经统计, 中国目前所知的石松类和硕类植物共计 2,244 种 (包括亚种和变种, 但不包括变型和杂交种)。评估结果为: 极危(CR) 43种(其中6种可能已经灭绝)、濒危(EN) 68种、 易危(VU) 71种、近危(NT) 66种、无危(LC) 1,124种、数据缺乏(DD) 872种。受威胁种类(包括VU、EN、CR)共计 182 种, 其中 79 种为中国特有或准特有种。特有而又极度濒危的植物有 24 种, 分别是梅山铁线䓲(Adiantum meishanianum)、荷叶铁线蕨(A. nelumboides)、秦氏莲座蕨(Angiopteris chingii)、壮乡铁角蕨(Asplenium cornutissimum)、 海南实蕨(Bolbitis hainanensis)、直叶金发石杉(Huperzia quasipolytrichoides var. rectifolia)、东方水非(Isoëtes orientalis)、台湾水非(I. taiwanensis)、云贵水韭(I. yunguiensis)、台湾曲轴蕨(Paesia taiwanensis)、海南金星蕨 (Parathelypteris subimmersa)、基羽鞭叶耳蕨(Polystichum basipinnatum)、洞生耳硕(P. cavernicola)、微小耳蒴( . minutissimum)、倒披针耳硕(P. oblanceolatum)、岩穴耳蒴(P. speluncicola)、长柄新月硕(Pronephrium longipetiolatum)、尾羽假毛蕨(Pseudocyclosorus caudipinnus)、罗浮牙蕨(Pteridrys lofouensis)、细叶凤尾蒴(Pteris angustipinna)、十字假瘤蕨(Selliguea cruciformis)、黑柄叉蒴(Tectaria ebenina)、河口叉蕨(T. hekouensis)和冈本氏岩蕨 (Woodsia okamotoi), 它们应予以最优先保护。目前的红色名录只是一个初步评估结果, 若要得到一份更完整更准 确的红色名录, 我们还需要做进一步的信息收集和评估工作, 特别要加强中国石松类和硕类植物的分类学研究。 今后评估工作中应重点关注的类群主要有莲座硕属(Angiopteris)、铁角硕属(Asplenium)、蹄盖硕属(Athyrium)、对

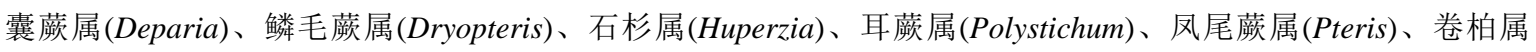
(Selaginella)、水龙骨科(Polypodiaceae)和金星蕨科(Thelypteridaceae), 这些类群的物种分布与生存信息目前最为缺乏。 关键词: 生物多样性; 保护; 石松类; 偋类植物; IUCN红色名录等级; IUCN红色名录标准; 受威胁种
\end{abstract}

\section{Red list assessment of lycophytes and ferns in China}

\author{
Shiyong Dong ${ }^{1 *}$, Zhengyu Zuo ${ }^{1,2}$, Yuehong Yan $^{3}$, Jianying Xiang ${ }^{4}$ \\ 1 Key Laboratory of Plant Resources Conservation and Sustainable Utilization, South China Botanical Garden, Chinese \\ Academy of Sciences, Guangzhou 510650 \\ 2 University of Chinese Academy of Sciences, Beijing 100049 \\ 3 Shanghai Chenshan Plant Science Research Center, Chinese Academy of Sciences; Shanghai Chenshan Botanical \\ Garden, Shanghai 201602 \\ 4 Yunnan Academy of Biodiversity, Southwest Forestry University, Kunming 650224
}

Abstract: To understand the extinction risk of lycophytes and ferns in China, we conducted an evaluation of the two groups according to IUCN Red List Categories and Criteria (version 3.1) at the national level. During a period of 16 months, a group of three researchers evaluated each taxon by means of reading literature, checking herbarium specimens, and consulting colleagues working on lycophytes and ferns in China. A total of 30 colleagues share their knowledge, mainly regarding living status and distribution, on Chinese lycophytes

收稿日期: 2016-07-24; 接受日期: 2016-10-27

基金项目：环境保护部“生物多样性保护专项”一中国高等植物红色名录; 国家标本资源共享平台一植物子平台(2005DKA21401)

* 通讯作者 Author for correspondence. E-mail: dongshiyong@scib.ac.cn 
and ferns. Here we present a preliminary red list of Chinese lycophytes and ferns which includes 2,244 taxa of the following categories: 43 Critically Endangered (CR, among which six have probably been already extinct) , 68 Endangered (EN), 71 Vulnerable (VU), 66 Near Threatened (NT), 1,124 Least Concern (LC), and 872 Data Deficient (DD). The taxa which have been taken into account include species, subspecies, and varieties only, with forms and hybrids excluded. Of the 182 threatened species (VU, EN, and CR), 79 are endemic (or nearly) to China. Conservation priorities are suggested for the 79 threatened and endemic species, especially the 24 critically endangered species which include Adiantum meishanianum, A. nelumboides, Angiopteris chingii, Asplenium cornutissimum, Bolbitis hainanensis, Huperzia quasipolytrichoides var. rectifolia, Isoëtes orientalis, I. taiwanensis, I. yunguiensis, Paesia taiwanensis, Parathelypteris subimmersa, Polystichum basipinnatum, P. cavernicola, P. minutissimum, P. oblanceolatum, P. speluncicola, Pronephrium longipetiolatum, Pseudocyclosorus caudipinnus, Pteridrys lofouensis, Pteris angustipinna, Selliguea cruciformis, Tectaria ebenina, T. hekouensis, and Woodsia okamotoi. This red list is of a preliminary nature as a rather high proportion of taxa belong to DD (up to 872, ca. 39 \% of total taxa in China). To gain a complete and precise red list, further evaluation work, especially taxonomic revisions based on field observations, is needed for the lycophytes and ferns in China. These include Angiopteris, Asplenium, Athyrium, Deparia, Dryopteris, Huperzia, Polystichum, Pteris, Selaginella, Polypodiaceae, and Thelypteridaceae which are still relatively poorly understood in terms of taxonomy and conservation.

Key words: biodiversity; conservation; lycophytes; ferns; IUCN Red List categories; IUCN Red List criteria; threatened species

石松类(lycophytes)和蕨类植物(ferns)是高等植 物的重要组成部分, 全球估计约13,600种, 其中石 松类约1,360种, 蕨类植物约12,240种(Moran, 2008), 二者都具较发达的维管组织, 并以孢子繁殖和配子 体可以独立生存等特征区别于种子植物。历史上石 松类长期被称作拟蕨类(fern allies), 并被认为是真 烣类(ferns)的近亲, 二者组成一个自然的分类群, 即蕨类植物门(Pteridophyta) (Tryon \& Tryon, 1982; 吴兆洪和秦仁昌，1991)或蕨类植物(pteridophytes) (Kramer \& Green, 1990)。但分子系统学的研究结果 表明, 石松类(包括石松科Lycopodiaceae, 水非科 Isoëtaceae, 卷柏科 Selaginellaceae) 是一个较早分化 的类群, 蕨类植物的姐妹群不是石松类而是种子植 物(spermatophytes) (Pryer et al, 2001; Smith et al, 2006)。中国的石松类和蕨类植物十分丰富, 约有 2,300种(张宪春, 2012), 约占世界已知总数的 $17 \%$ 。 近年出版的Flora of China记载了2,129种石松类和 烣类植物, 分属于 38 科117属(Wu et al, 2013)。最近 另有统计为 40 科178属2,147种(严岳鸿等, 2016)。

自20世纪80年代以来，中国石松类和蕨类植物 多样性的保护, 即为中国学术界和政府部门所关 注。比如荷叶铁线蕨(Adiantum nelumboides, 曾用名 A. reniforme var. sinense)、对开鄀(Asplenium komarovii, 曾用名 Phyllitis scolopendrium)、杪椤 (Alsophila spinulosa)等13种蕨类植物(广义, 包含石松类)先后
被列入《中国珍稀濒危保护植物名录》(宋朝枢等, 1989)和《中国植物红皮书》(傅立国和金鉴明, 1992); 光叶蕨(Cystopteris chinensis, 曾用名Cystoathyrium chinense)、单叶贯众(Cyrtomium hemionitis)、中华水 非(Isoëtes sinensis)和毛脉蕨(Trichoneuron microlepioides)被评估为中国已灭绝的种类, 而尖叶卤蕨 (Acrostichum speciosum) 等101种被认为是中国濒危 或稀有种的代表(《中国生物多样性国情研究报告》 编写组, 1998); 至1999年, 杪椤科、蛙壳䓞科、水非 属(Isoëtes)、水蕨属(Ceratopteris)的全部种类, 以及 法斗观音座莲(Angiopteris sparsisora)等14种蕨类植 物被列入“国家重点保护野生植物名录(第一批)” (于永福, 1999; 张宪春, 2013)。近年由日本学者牵 头发表了一个狭域分布的“亚洲珍稀和受威胁䓲类 植物名录” (Ebehara et al, 2012), 收录了886个种及 种下分类群，其中144种为中国个别或少数省区有 分布的石松类和蕨类植物。这些珍稀濒危或重点保 护名录, 几乎都是依据经验评估而得出的, 并未采 用统一的标准进行严格评估。

为了规范评价物种的濒危等级, 自1993年起, 世界自然保护联盟物种生存委员会(IUCN/SSC)制 定并不断完善了物种红色名录濒危等级体系和评 价标准(IUCN, 1993，2001，2003; IUCN Standards and Petitions Subcommittee, 2010)。IUCN红色名录 等级和标准是一个相对客观的评价物种濒危程度 
的工具, 被世界各地学者广为接受。亚洲区域内, 各国学者最近十多年以来也陆续开始采用IUCN红 色名录等级和标准评估石松类和蕨类植物的濒危 等级, 涉及的具体地域包括中国海南岛(董仕勇等, 2003; 董仕勇, 2004, 2013)、中国台湾岛(Wang et al, 2012)、中国全境(严岳鸿等，2013)、日本(Japanese Ministry of Environment, 2007)、菲律宾(Fernando et $\mathrm{al}, 2008$ )、马来半岛(Parris et al, 2010)、印度尼西亚 的西巴布亚省(Johns et al, 2006)、以及中南半岛的部 分地区(泰国、老挝和柬埔寨) (Lindsay \& Middleton, 2012)。为了更准确地了解中国石松类和偋类植物的 生存现状和濒危等级, 自2009年开始, 我们开展了 历时一年又四个月的中国石松类和蕨类植物的 IUCN 红色名录等级评估, 以期为优先保护一些濒 危和极度濒危的石松类和蕨类植物提供科学决策 的依据。

\section{1 评估方法及评估过程}

\section{1 评估对象和分类群的名称}

评估对象为中国原生的全部石松类和葓类植 物, 2009年主要依据《中国植物志》2-6卷统计为 2,423种(包括种下等级), 当时的科属概念主要采用 1978年秦仁昌系统。近年来随着分子系统学研究的 深入, 蕨类植物本身的定义及大量科属的概念发生 了改变。2013年出版的Flora of China (FOC)的石松 类和硕类卷册(Wu et al, 2013)采用了一个全新的分 类系统, 许多物种的名称发生了变化。本文所提及 的石松类和蒝类的科属概念, 均采纳FOC分类系统 的概念, 分类群的名称也遵循FOC所采用的名称 (极少数名称有更新)。

\section{2 评估依据和评估等级}

评估依据为IUCN Red List Categories and Criteria, Version 3.1 (IUCN, 2001)和Guidelines for Using the IUCN Red List Categories and Criteria, Version 4.0. (IUCN Standards and Petitions Subcommittee, 2010)。IUCN红色名录等级共包含未评估 (Not Evaluated, NE)、数据缺乏(Data Deficient, DD)、 无危(Least Concern, LC)、近危(Near Threatened, NT)、易危(Vulnerable, VU)、濒危(Endangered, EN)、 极危(Critically Endangered, CR)、野外灭绝(Extinct in the Wild, EW)和灭绝(Extinct, EX)等8类。

\section{3 评估过程}

物种红色名录的评估经历了如下 5 个步骤:

第一步, 名单初䇻。2009年9月开始, 根据已掌 握的文献和以往野外调查资料, 将最初统计的 2,423种中国石松类和䓲类植物初步划分为 3 类: 无 危类(882种)、数据缺乏类 $(1,210$ 种) 和拟重点评估类 (331种)。其中, 拟重点评估类是推测可能濒危并准 备重点评估的种类, 入选标准包括: 以往文献中记 载的濒危种类、系统位置独特的种类、特殊生境中 的植物、以及有重要经济价值的种类。

第二步, 评估信息集中征求。2010年4月开始, 我们把331种待重点评估的物种名单, 分发给32位 蕨类同行, 请求填写并反馈评估所需的信息(表格 形式)。至2010年7月5日, 收到其中15位专家的反馈, 反馈信息覆盖92种。表格回收成功率近50\% (15/32), 有效信息约 $92 \%(75 / 92)$ 。

第三步, 重点评估。针对拟重点评估的物种展 开评估。评估小组成员(董仕勇、严岳鸿和向建英) 按类群或区系熟悉程度分工: 董仕勇主要评估热带 类群, 严岳鸿主要评估华中地区(亚热带)的种类, 向建英评估鳞毛偋属(Dryopteris)的种类。至2010年 11月底, 重点评估初步完成。

具体评估过程包括信息汇总(各个渠道的标本 信息、野外调查信息及文献资料)、逐条比对IUCN 红色名录等级与标准、确定等级、填写评估说明。 在具体评估过程中, 针对不同类群设计信息调查表, 通过电话和邮件向多位同行征询物种的居群信息。

第四步, 初步篮选的数据缺乏(DD)类的再评 估。针对最初筛选为数据缺乏(DD)的 1,210 种石松类 和硕类植物，设计简易的信息调查表，把其中的 913种发给17位硕类同行, 请求提供物种信息。至 2010年11月底, 共有10位同行返回调查表, 提供了 469种蕨类植物的简易居群信息。

第五步, 评估信息汇总与统计。2010年12月完 成。2016年1月, 改用FOC所采用的分类系统, 重新 统计中国石松类和蕨类植物IUCN红色名录各等级 的数目, 补充评估新增加的种类, 完成评估报告。

\section{2 结果}

据《中国植物志》偋类卷册和Flora of China 2-3 卷所记载的种类进行统计, 中国石松类和硕类植物 共计 2,244种(包括6个亚种和 119 个变种, 不计种下 
变型和杂交种), 其中43种极危(CR), 68种濒危(EN), 71种易危(VU), 66种近危(NT), 1,124种无危(LC), 872种属于数据缺乏(DD) 的种类。受威胁种类包括 易危(VU)、濒危(EN)和极危(CR), 共计182种, 其中

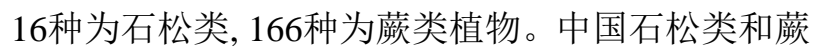
类植物各濒危等级物种数及所占比例见图1。

\section{1 受威胁种类}

182 个受威胁种类分属于 28 科 87 属, 主要集中 在鳞毛偋科(Dryopteridaceae, 31种)、水龙骨科 (Polypodiaceae, 26种)、凤尾厥科(Pteridaceae, 18种)、 石松科(Lycopodiaceae, 13种)、蹄盖痥科(Athyriaceae, 13种)、膜蕨科(Hymenophyllaceae, 12种)和叉䓲科 (Tectariaceae, 10 种)等7个科中。这些被评估为受威 胁的种类, 多数都是因为生境被破坏或受干扰, 物 种的种群大小正在不断衰减之中。如云贵水非 (Isoëtes yunguiensis), 中国特产, 曾在云南(昆明、寻 甸等地)和贵州(平坝)有分布记录, 生长在中高海拔 山区的沼泽地带(庞新安等, 2003), 但最近的调查资 料显示, 该种仅贵州平坝居群仍然存活, 最近数年 间, 由于人类活动和其他植物的强势竞争, 其适宜 生境不断缩减, 现存居群的个体总数不足 50 株, 居 群减小达 $80 \%$ 以上(陈进明等, 2005), 其受威胁等级 达到极危级(CR)。少部分物种被评估为受威胁主要 是考虑到其种群太小, 中国种群消失或物种灭绝的 风险较大。比如海南蹄盖葓(Athyrium hainanense), 这是一个海南特有种, 只在海南五指山和鹦哥岭有

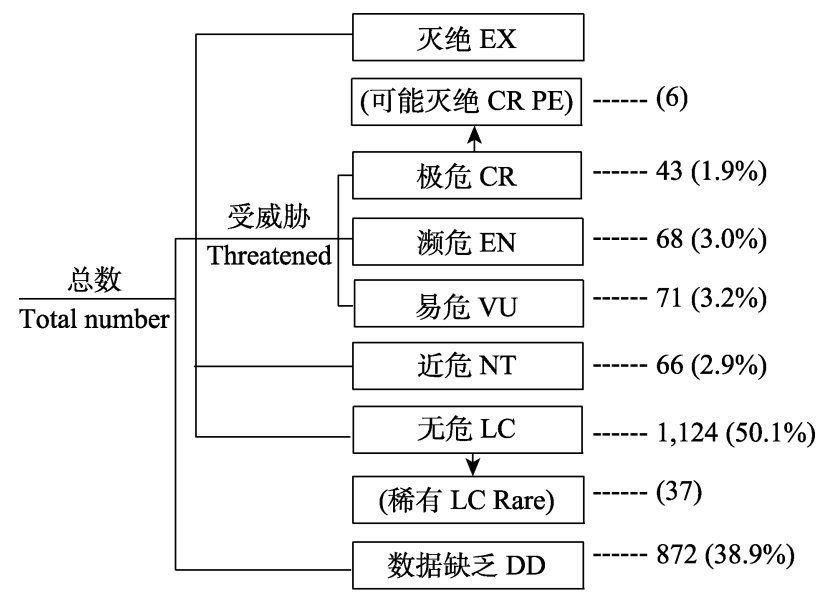

图1 中国石松类和莿类植物红色名录等级及各等级所占的 比例

Fig. 1 Red List of Chinese lycophytes and ferns: the composition (categories and number of taxa in each category) and their proportions
分布, 见于热带山地雨林或山地灌从中, 土生; 五 指山居群很小, 估计不足 50 株, 近年来受游客活动 的严重干扰可能已经消失; 鸭哥岭居群稍大, 估计 在200株左右，目前尚没有显著的威胁因素。鉴于该 种的成熟个体总数可能不足 250 株, 所以将其划入 濒危(EN)。

光叶偋、单叶贯众、中华水非毛脉疹等 4 种 植物曾经被评估为中国区域内灭绝(《中国生物多样 性国情研究报告》编写组, 1998)。现在的评估结果 为, 除毛脉葓的资料缺乏(DD类)外, 其他 3 种都在 濒危(EN)之列。最新调查资料显示, 光叶硕目前尚 存一个很小的居群, 生于常绿落叶阔叶混交林下, 个体数量为 58 株, 干扰威胁相对较低(余凌帆等, 2015)。中华水非和单叶贯众属于濒危类。中华水非 在湖南、安徽、浙江等省区还有分布(庞新安等, 2003), 而单叶贯众至少还有广西和云南的居群(个 人调查资料)。

杪椤科因起源古老(起源于晚侏罗纪)和树形外 貌(现存硕类植物中很少能长成乔木状)而备受关注, 中国全部杪椤科植物均被列为国家II类重点保护植 物(于永福, 1999)。对照IUCN红色名录标准, 中国有 分布的 14 种杪椤科植物中, 多数种类(9种)被评估为 无危(LC), 受威胁的有3种, 即毛叶杪椤 (Alsophila andersonii) 为濒危( $\mathrm{EN}$ ), 滇南杪椤 (A. austroyunnanensis)和兰屿杪椤 (A. fenicis)为易危 (VU)。此外, 喀 西杪椤(A. khasyana)的居群信息缺乏(DD); 南洋杪 椤(A. loheri)目前仅有 1 个分布点, 个体数量也不多, 被评估为近危(NT) (Wang et al, 2012)。

43 种极危 $(\mathrm{CR})$ 物种中, 有 6 种可能已经灭绝或 在中国境内已经灭绝, 即针叶硕 (Monogramma trichoidea)、尾羽假毛䓲(Pseudocyclosorus caudipinnus)、海南实蕨(Bolbitis hainanensis)、罗浮牙蕨 (Pteridrys lofouensis)、多变叉䓲(Tectaria variabilis) 和十字假瘤蒝(Selliguea cruciformis)。除针叶硕有较 大的分布区外, 另外 5 种都是狭域分布种, 最近数 十年间中国范围内未见采集记录, 这6种植物的具 体分布和采集信息列于表 1 。

\section{2 近危 $(\mathrm{NT})$ 种类}

66种近危种类中, 包括 9 种石松类和 57 种偋类 植物, 分属于 17 科 38 属, 以水龙骨科和凤尾茨科的 植物居多。对于大部分近危植物而言, 它们在中国 的分布区域并不十分狭小, 但种群小, 个体数量不 
表1 6种在中国可能已经灭绝的疹类植物

Table 1 Six fern species evaluated to be probably extinct in China

\begin{tabular}{|c|c|}
\hline 种 Species & 分布和采集信息 Distribution and collection information \\
\hline $\begin{array}{l}\text { 海南实萨 } \\
\text { Bolbitis hainanensis }\end{array}$ & $\begin{array}{l}\text { 中国特产(海南和云南), 海南仅有1份1933年的凭证标本, 具体采集地不详; 云南仅有1956年采自思茅的3号 } \\
\text { 标本, 之后再未见有采集记录。Endemic to China (in Hainan and Yunnan). There is only one collection from } \\
\text { Hainan in } 1933 \text { and three collections from Yunnan in 1956. It has not been collected since } 1956 \text {. }\end{array}$ \\
\hline $\begin{array}{l}\text { 针叶藏 } \\
\text { Monogramma trichoidea }\end{array}$ & $\begin{array}{l}\text { 分布于中国(海南和台湾)及亚洲热带地区, 附生于热带雨林林下石上。台湾只有1923年的采集凭证; 海南仅 } \\
\text { 有1935年的1份采集记录, 最近数十年来, 海南热带低地森林开发严重, 估计该种在海南已经消失。 } \\
\text { Distributed in Hainan and Taiwan of China and other tropical regions of Asia, lithophytic in rain forest. There is } \\
\text { only one voucher from Taiwan in } 1923 \text { and one from Hainan in 1935, respectively. It is likely extinct in Hainan } \\
\text { due to the heavy deforestation in past several decades. }\end{array}$ \\
\hline $\begin{array}{l}\text { 尾羽假毛萨 } \\
\text { Pseudocyclosorus caudipinnus }\end{array}$ & $\begin{array}{l}\text { 海南特有, 但迄今只有 } 1 \text { 份 } 1878 \text { 年的模式标本。Endemic to Hainan. It is represented only by its type specimen } \\
\text { collected in } 1878 .\end{array}$ \\
\hline $\begin{array}{l}\text { 罗浮牙蕨 } \\
\text { Pteridrys lofouensis }\end{array}$ & $\begin{array}{l}\text { 中国特产(贵州和云南), 至今只有3份凭证标本, 其中 } 1 \text { 份为模式标本, } 1910 \text { 年以前采自贵州罗甸; 另2份标本 } \\
\text { 分别于1954年采自云南屏边和1985年采自云南西畴。我们推测该种已经绝灭, 但没有针对该种开展过详细 } \\
\text { 的调查, 尚不十分肯定。Endemic to China (in Guizhou and Yunnan), only three collections, with one (the type) } \\
\text { from Luodian, Guizhou collected before } 1910 \text { and two from Yunnan (Pingbian in } 1954 \text { and Xichou in 1985). This } \\
\text { species is likely extinct and we are not sure because of no special data available. }\end{array}$ \\
\hline $\begin{array}{l}\text { 十字假瘤蕨 } \\
\text { Selliguea cruciformis }\end{array}$ & $\begin{array}{l}\text { 分布于中国(广东)、越南北部和泰国北部。中国范围内, 历史上仅有 } 1 \text { 份 } 1924 \text { 年的模式标本, 采自广东北部龙 } \\
\text { 头山, 此后再没有该种在中国的记录。Distributed in China (in Guangdong), northern Vietnam and northern } \\
\text { Thailand. There is only one record from China, i.e., the type specimen from Longtoushan, northern Guangdong in } \\
\text { 1924. Since then there is no any records of this species in China. }\end{array}$ \\
\hline $\begin{array}{l}\text { 多变叉硕 } \\
\text { Tectaria variabilis }\end{array}$ & $\begin{array}{l}\text { 分布于中国(海南)和越南。中国的采集记录只有 } 1935 \text { 年的 } 1 \text { 份凭证标本，此后再没有该种的采集记录。 } \\
\text { Distributed in China (in Hainan) and Vietnam. There is only one collection known in China which was gathered in } \\
1935 .\end{array}$ \\
\hline
\end{tabular}

多, 所处的生境容易受外界干扰。其中不少物种的 种群大小正处于缩减之中, 如果干扰因素得不到有 效控制, 预期不远的将来, 它们很有可能达到易危 (VU)的标准。比如中华石杉(Huperzia chinensis), 特 产中国陕西、湖北、四川等地, 生于中高海拔地区 草坡和岩石缝中, 调查资料显示, 该种的栖息地质 量近年来明显退化, 分布面积减小近 $50 \%$, 估计种 群大小减小近 $30 \%$, 接近易危(VU)的评估标准。

\section{3 无危 $(\mathrm{LC})$ 种类}

1,124种无危类中，包括 63 种石松类和 1,061 种 硕类植物, 对照IUCN的标准, 它们目前的生存状 态相对安全, 无须担心其种群会有减小或灭绝的风 险。它们中的大多数是常见种类, 但有 37 种是狭域 分布的种, 中国范围内通常只见于1-3个省区 (未计 新近发表的新种), 其中14种为中国特有成分。稀有 但暂无生存威胁的14种中国特有种分别是高寒水 非(Isoëtes hypsophila)、海南卷柏(Selaginella hainanensis)、海南鳞始蕨(Lindsaea hainaniana)、广东 凤尾硕(Pteris guangdongensis)、黄志双盖葓(Diplazium wangii)、角状耳蒝(Polystichum alcicorne)、峨 眉耳蕨(P. caruifolium)、海南复叶耳疹(Arachniodes hainanensis)、黑鳞复叶耳蕨(A. nigrospinosa)、海南 节毛蕨(Lastreopsis subrecedens)、中华舌硕(Elaphog- lossum sinii)、网藤蕨(Lomgramma matthewii)、海南 瓦韦(Lepisorus affinis)和扇硕(Neocheiropteris palmatopedata)。

\section{4 数据缺乏(DD)种类}

872种数据缺乏类中, 包括 60 种石松类和 812 种 蕨类植物，其中种类最多的 5 个科分别是鳞毛䓲科 (223种, 占科内全部种数的 $44.8 \%$ )、蹄盖疹科(177 种, 55.5\%)、金星嵚科 (Thelypteridaceae) (89种， $42.2 \%$ )、凤尾蕨科(81种，30.6\%)和水龙骨科(78种， $28.6 \%)$ 。属级水平上, 评估信息缺乏的主要是耳濒 属(Polystichum) (130种, 占属内全部种数的63\%)、 蹄盖蒝属(Athyrium) (86种, 64.2\%)、鳞毛烣属(53种,

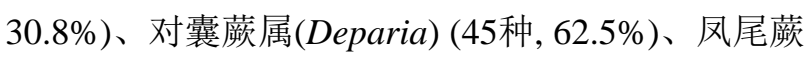
属(Pteris) (41种, 42.7\%)和双盖硕属(Diplazium) (37 种, $38.1 \%)$ 。

\section{3 讨论}

此次评估是集全国蕨类同行之力(综合目前中 国蕨类同行的知识和资料积累), 由受训练的评估 团队, 整合了最新的分类学资料和调查研究成果, 严格按照IUCN的评估流程而开展的国家水平上的 红色名录评估。这一评估结果一定程度上反映了当 前中国石松类和䓲类植物的生存和濒危现状。此前 
列入“珍稀濒危保护植物名录” (宋朝枢等，1989)或 《中国植物红皮书: 稀有濒危植物》(傅立国和金鉴 明, 1992)的13种石松类和蒝类植物, 现在有了更为 准确的红色名录等级或濒危等级。曾经认为已经易 危或濒危的原始观音座莲(现在的接受名是Angiopteris latipinna)、杪椤、扇蕨(Neocheiropteris palmatopedata), 按IUCN红色名录标准评估, 都属于无 危(LC)类, 目前相对安全, 并未面临较大的灭绝风 险。曾经评估为濒危的荷叶铁线蒝、云贵水非【长 期错误鉴定为宽叶水非(I. japonica) (Zhang \& Taylor, 2013)】、以及被认为是稀有的鹿角偋(Platycerium wallichii), 最新的评估结果表明, 它们都是极 危(CR)类, 面临较大的生存压力。

另一方面, 本研究所获得的红色名录仍然是一 个阶段性的评估结果，其中包含较高比例(约39\%) 的DD类(数据缺乏), 我们对这些植物的真正的分类 学地位、在中国的地理分布、居群大小和生境现状 还所知甚少, 很难依据评估标准赋予一个红色名录 等级。另外, 对于部分甚至较高比例的评估为LC及 以上等级(NT, VU, EN, CR)的物种而言, 目前评估 所赋予的等级可能与实际情况并不相符。因为此次 评估所依赖的信息，主要来自于文献、标本和各位 同行在平时工作、特别是野外做分类或区系调查时 所积累的资料, 很少去针对某一类群或某个物种开 展详细的野外调查, 所以很难准确获取评估所必需 的信息, 从而保证评估结果确切可信。想要获取一 份更完整准确的红色名录, 还需要做进一步的信息 收集和评估工作。

物种的受威胁等级并不是一成不变的。物种的 红色名录等级有一定的时效性, 目前的评估结果只 代表现在(或者更准确地说是5年以前)的濒危状态。 一次评估所得到的等级, 只反映物种在评估时期所 处的生存状态，随着时间和所处环境条件的改变, 其受威胁等级很可能会发生变化。因此, 等级之间 应该保持一个动态的变化, 物种的红色名录等级绝 对不是一成不变的。IUCN建议, 某一物种如果5年 以上不再符合较高等级的标准, 就应当降低等级 (IUCN, 2001; IUCN Standards and Petitions Subcommittee, 2010)。为了及时更新物种的红色名录等级, 也便于及时更改评估错误, 从而更及时有效地保护 生物多样性，应定期开展物种红色名录的再评估。

从保护物种多样性的角度考虑, 只有中国特有
或准特有(主产中国, 也有部分居群生长在邻国)且 又受威胁的石松类和蕨类植物才应当优先保护。也 就是说，受威胁等级与优先保护之间并不是严格对 应的。因为此次的评估是地区水平(中国范围内)、 而不是全球水平的评估。一个在中国范围内属于极 危 (CR) 的物种, 在全球范围内可能是无危的 (LC 类)。比如二歧莎草蒝(Schizaea dichotoma), 这是一 个旧大陆热带和亚热带地区广泛分布的种, 它的主 要分布区在南半球的澳洲及太平洋岛屿, 中国南部 地区(海南)是其边缘分布区(Chinnock，1998; Zhang \& Mickel, 2013)。二歧莎草欮虽然在海南的种群极 小, 生存面临威胁, 但并不应列为中国重点保护植 物, 因为即使它在中国范围内灭绝, 也不影响它的 全球种群大小。反之, 如果一个中国特有种在中国 范围内灭绝, 就意味着我们的星球将会损失一个物 种。所以, 受威胁的中国特有种和准特有种才是我 们应当优先保护的对象。目前评估的中国182种受 威胁石松类和疹类植物中, 有 79种为中国特有或 准特有种(表2), 建议中国政府和学术界考虑优先 保护这79种植物, 特别是其中已经极危(部分可能 已经灭绝)的 24 种, 即梅山铁线偋 (Adiantum meishanianum)、荷叶铁线硕、秦氏莲座萩(Angiopteris chingii)、壮乡铁角葓(Asplenium cornutissimum)、 海南实薑、直叶金发石杉(Huperzia quasipolytrichoides var. rectifolia)、东方水非(Isoëtes orientalis)、台湾水 非(I. taiwanensis)、云贵水韭、台湾曲轴䕬(Paesia taiwanensis)、海南金星蒴(Parathelypteris subimmersa)、基羽鞭叶耳蒴(Polystichum basipinnatum)、 洞生耳蕨 (P. cavernicola)、微小耳䓲(P. minutissimum)、倒披针耳蕨( $P$. oblanceolatum)、岩穴耳蕨 (P. speluncicola)、长柄新月蕨(Pronephrium longipetiolatum)、尾羽假毛蕨、罗浮牙蕨、细叶凤尾硕 (Pteris angustipinna)、十字假瘤蒴、黑柄叉锁(Tectaria ebenina)、河口叉䓲(T. hekouensis)和冈本氏岩 䕬(Woodsia okamotoi)。

当然, 中国受威胁的石松类和硕类植物应该 远远不止182种, 因为尚有大量的(872种)物种数据 缺乏(DD), 我们对这些物种的了解还非常有限。就 我们的评估经历而言, 难以评估的类群往往其分 类学研究比较薄弱, 而此次得到较准确评估的类 群, 则是分类学研究, 尤其是野外工作比较细致深 入的类群，比如叉硕属(Tectaria)、铁角蕨属巢蒝群 
表2 中国特有或准特有且受威胁的石松类和簌类植物。CR: 极度濒危; EN: 濒危; VU: 易危。*指示也分布到越南北部。

Table 2 A list of threatened and meanwhile endemic or nearly endemic lycophytes and ferns in China. CR, Critically Endangered; EN, Endangered; VU, Vulnerable. The asterisk (*) indicates species with distribution also in northern Vietnam.

\begin{tabular}{|c|c|c|c|c|c|}
\hline \multicolumn{2}{|l|}{ 分类群 Taxon } & \multirow[t]{2}{*}{$\begin{array}{l}\text { 等级 } \\
\text { Category }\end{array}$} & \multicolumn{2}{|l|}{ 分类群 Taxon } & \multirow[t]{2}{*}{$\begin{array}{l}\text { 等级 } \\
\text { Category }\end{array}$} \\
\hline 石松类 Lycophyte & & & & & \\
\hline 皱边石杉 & Huperzia crispata & VU & 独山双盖蕨 & Diplazium dushanense & VU \\
\hline 直叶金发石杉 & Huperzia quasipolytrichoides var. rectifolia & CR & 马鞍山双盖蕨 & Diplazium maonense & VU \\
\hline 东方水非 & Isoëtes orientalis & $\mathrm{CR}$ & 深裂双盖蒴 & Diplazium metcalfii & VU \\
\hline 中华水非 & Isoëtes sinensis & EN & 四棱双盖硕 & Diplazium quadrangulatum & VU \\
\hline 台湾水非 & Isoëtes taiwanensis & CR & 网脉双盖蕨 & Diplazium stenochlamys* & VU \\
\hline 云贵水非 & Isoëtes yunguiensis & CR & 大平鳞毛䕬 & Dryopteris bodinieri* & EN \\
\hline 棣氏卷柏 & Selaginella devolii & EN & 两广鳞毛䕬 & Dryopteris liangkwangensis* & EN \\
\hline 琉球卷柏 & Selaginella lutchuensis & EN & 黄山鳞毛蕨 & Dryopteris whangshangensis & EN \\
\hline 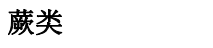 & ferns & & 琼崖舌蕨 & Elaphoglossum luzonicum var. mcclurei & VU \\
\hline 台湾铁线偋 & Adiantum formosanum & VU & 心叶薄唇偋 & Leptochilus cantoniensis* & VU \\
\hline 仙霞铁线硕 & Adiantum juxtapositum & VU & 碎叶鳞始蕨 & Lindsaea chingii* & EN \\
\hline 梅山铁线偋 & Adiantum meishanianum & CR & 中华藤薜 & Lomariopsis chinensis & VU \\
\hline 荷叶铁线蕨 & Adiantum nelumboides & CR & 台湾曲轴蕨 & Paesia taiwanensis & CR \\
\hline 台湾高山铁线蕨 & Adiantum roborowskii var. taiwanianum & VU & 海南金星鰀 & Parathelypteris subimmersa & CR \\
\hline 黑柄粉背蕨 & Aleuritopteris ebenipes & VU & 广东马尾杉 & Phlegmariurus guangdongensis & VU \\
\hline 中国蕨 & Aleuritopteris grevilleoides & VU & 台湾马尾杉 & Phlegmariurus taiwanensis & VU \\
\hline 毛叶粉背薜 & Aleuritopteris squamosa & EN & 节毛耳䓲 & Polystichum articulatipilosum & EN \\
\hline 滇南杪椤 & Alsophila austroyunnanensis & VU & 基羽鞭叶耳眇 & Polystichum basipinnatum & CR \\
\hline 二回莲座薜 & Angiopteris bipinnata & EN & 洞生耳蕨 & Polystichum cavernicola & CR \\
\hline 秦氏莲座蕨 & Angiopteris chingii & CR & 卵状边叶耳粆 & Polystichum conjunctum & VU \\
\hline 相马氏莲座蕨 & Angiopteris somae & EN & 广东耳偋 & Polystichum kwangtungense & VU \\
\hline 四回毛枝蕨 & Arachniodes sinomiqueliana & EN & 微小耳偋 & Polystichum minutissimum & CR \\
\hline 壮乡铁角偋 & Asplenium cornutissimum & CR & 倒披针耳薜 & Polystichum oblanceolatum & CR \\
\hline 黑边铁角蕨 & Asplenium speluncae & EN & 岩穴耳偋 & Polystichum speluncicola & CR \\
\hline 长叶蹄盖偋 & Athyrium elongatum & VU & 天坑耳蕨 & Polystichum tiankengicola & EN \\
\hline 海南蹄盖鰀 & Athyrium hainanense & EN & 长柄新月偋 & Pronephrium longipetiolatum & $\mathrm{CR}$ \\
\hline 小蹄盖蕨 & Athyrium minimum & EN & 尾羽假毛蕨 & Pseudocyclosorus caudipinnus & CR \\
\hline 启无蹄盖蕨 & Athyrium wangii & VU & 罗浮牙偋 & Pteridrys lofouensis & CR \\
\hline 海南实鄀 & Bolbitis hainanensis & $\mathrm{CR}$ & 细叶凤尾蕨 & Pteris angustipinna & CR \\
\hline 高平蕨 & Caobangia squamata* & VU & 十字假瘤蕨 & Selliguea cruciformis* & CR \\
\hline 边果蕨 & Craspedosorus sinensis & EN & 大围山假瘤蕨 & Selliguea daweishanensis & VU \\
\hline 滇桂肋毛鄀 & Ctenitis dianguiensis & EN & 圆顶假瘤蕨 & Selliguea obtusa & VU \\
\hline 焕镛钩毛蕨 & Cyclogramma chunii & EN & 屏边溪边蕨 & Stegnogramma dictyoclinoides* & EN \\
\hline 秦氏贯众 & Cyrtomium chingianum & EN & 天长罗曼偋 & Struthiopteris eburnea var. obtusa & VU \\
\hline 惠水贯众 & Cyrtomium grossum & EN & 黑柄叉瞢 & Tectaria ebenina* & CR \\
\hline 邢氏贯众 & Cyrtomium shingianum & EN & 粗齿叉蕨 & Tectaria grossedentata & EN \\
\hline 新宁贯众 & Cyrtomium sinningense & EN & 河口叉蕨 & Tectaria hekouensis & CR \\
\hline 台湾贯众 & Cyrtomium taiwanianum & EN & 五裂叶叉䕬 & Tectaria quinquefida* & EN \\
\hline 光叶鄀 & Cystopteris chinensis & EN & 海南符藤颐 & Teratophyllum hainanense* & EN \\
\hline 海南对囊偋 & Deparia hainanensis & EN & 冈本氏岩偋 & Woodsia okamotoi & $\mathrm{CR}$ \\
\hline
\end{tabular}

(Asplenium nidus group)。因此, 基于野外调查和标 本形态的严谨的分类学修订工作极其重要并且极 为紧迫。我们呼吁中国学界要大力加强分类学研究, 特别要针对评估信息缺乏较多的金星葓科、水龙骨
科, 以及耳蒴属、蹄盖蕨属、鳞毛蕨属、对囊蕨属、 凤尾硕属、铁角彍属(Asplenium)、卷柏属(Selaginella)、莲座董属(Angiopteris)、石杉属(Huperzia)等 类群开展详细的野外调查和基于形态性状研究的 
分类学修订工作。

致谢：评估过程中承蒙下列同行提供物种信息：陈 进明、陈珍传、丁炳扬、郭晓思、郭治友、何海、 和兆荣、蒋日红、李策宏、李中阳、刘保东、刘红 梅、刘全儒、刘以诚、卢金梅、陆树刚、齐新萍、 郡文、宋晓卿、王发国、王玛丽、徐成东、杨东梅、 杨鲁红、詹选怀、张钢民、张宪春、张艺翰、朱圣 朝、曾汉元。中国科学院植物研究所覃海宁博士的 科研团队为本项目的评估提供了大量的数据与技 术支持。在此一并致谢!

\section{参考文献}

Chen JM, Liu X, Wang QF (2005) Genetic diversity in Isoëtes yunguiensis, a rare and endangered endemic fern in China. Journal of Wuhan University (Natural Science Edition), 51, 767-770. (in Chinese with English abstract) [陈进明, 刘星, 王青锋 (2005) 中国珍稀特有濒危蒝类植物一一云贵水 非的遗传多样性. 武汉大学学报 (理学版), 51, 767-770.]

Chinnock RJ (1998) Schizaeaceae. In: Flora of Australia, Vol. 48, Ferns, Gymnosperms and Allied Groups (ed. McCarthy PM), pp. 177-183. ABRS/CSIRO Australia, Melbourne.

Compilation Group of China's Biodiversity: A Country Study (1998) China's Biodiversity: A Country Study. China Environmental Science Press, Beijing. (in Chinese) [《中国 生物多样性国情研究报告》编写组 (1998) 中国生物多样 性国情研究报告. 中国环境科学出版社, 北京.]

Dong SY (2004) Classification, Phytogeography and Conservation of the Pteridophytes from Hainan Island. PhD dissertation, Institute of Botany, Chinese Academy of Sciences, Beijing. (in Chinese with English abstract) [董仕 勇 (2004) 海南岛蕨类植物的分类、区系地理与保育. 博 士学位论文, 中国科学院植物研究所, 北京.]

Dong SY (2013) A revised checklist and assessment of pteridophytes, based on IUCN Red List Criteria. In: Biodiversity and Conservation of Hainan Yinggeling Nature Reserve (eds Jiang HS, Chan BPL, Zhou YD, Wang CD, Fang L, Lo YFP), pp. 51-67. China Forestry Publishing House, Beijing. (in Chinese with English abstract) [董仕勇 (2013) 硕类植物名录更新与濒危状况评估. 见: 海南鹦 哥岭自然保护区生物多样性及其保育(江海声, 陈辈乐, 周亚东, 王春东, 方林, 罗益奎 等编著), 51-67页. 中国 林业出版社, 北京.]

Dong SY, Chen ZC, Zhang XC (2003) Biodiversity and conservation of pteridophytes from Diaoluo Mountain, Hainan Island. Biodiversity Science, 11, 422-431. (in Chinese with English abstract) [董仕勇, 陈珍传, 张宪春 (2003) 海南岛吊罗山莿类植物的多样性及其保育. 生物 多样性, 11, 422-431.]

Ebehara A, Fraser-Jenkins C, Parris BS, Zhang XC, Yang YH,
Chiou WL, Chang HM, Lindsay S, Middleton D, Kato M, Praptosuwiryo TN, Amoroso VB, Barcelona JF, Ranil RHG, Park CH, Murakami N, Hoya A (2012) Rare and threatened pteridophytes of Asia 1. An enumeration of narrowly distributed taxa. Bulletin of the National Science Museum, Tokyo. Ser. B, Botany, 38, 93-119.

Fernando ES, Co LL, Lagunsad DA, Gruezo WSM, Barcelona JF, Madulid DA, Lapiz AB, Texon GI, Manila AC, Zamora PM (2008) Threatened plants of the Philippines: a preliminary assessment. The Asian International Journal of Life Sciences (Suppl. 3), 1-52.

Fu LK, Jin JM (1992) Chinese Plant Red Data Book (Vol. 1): Rare and Endangered Plants. Science Press, Beijing. (in Chinese) [傅立国, 金鉴明 (1992) 中国植物红皮书: 稀有 濒危植物 (第一册). 科学出版社, 北京.]

IUCN (1993) Draft IUCN Red List Categories. IUCN, Gland, Switzerland.

IUCN (2001) IUCN Red List Categories and Criteria, Version 3.1. IUCN Species Survival Commission. IUCN, Gland, Switzerland and Cambridge, UK.

IUCN (2003) Guidelines for Application of IUCN Criteria at Regional Levels, Version 3.0. IUCN Species Survival Commission. IUCN, Gland, Switzerland and Cambridge, UK.

IUCN Standards and Petitions Subcommittee (2010) Guidelines for Using the IUCN Red List Categories and Criteria, Version 4.0. http://www.iucnredlist.org/documents/reg_guidelines_en.pdf (accessed on 2016-01-10)

Japanese Ministry of Environment (2007) Red List of Japan, Plant I (Vascular plants). http://www.env.go.jp/press/file_view.php?serial=9947\&hou_id=8648 (accessed on 2016-01-10)

Johns RJ, Edwards PJ, Utteridge TMA, Hopkins HCF (2006) A Guide to the Alpine and Subalpine Flora of Mount Jaya. Royal Botanic Gardens, Kew, London.

Kramer KU, Green PS (1990) The Families and Genera of Vascular Plants, Pteridophytes and Gymnosperms, Vol. 1. Springer-Verlag, Berlin.

Lindsay S, Middleton D (2012 onwards) Ferns of Thailand, Laos and Cambodia. http://rbg-web2.rbge.org.uk/thaiferns/ (accessed on 2016-01-10)

Moran RC (2008) Diversity, biogeography, and floristics. In: Biology and Evolution of Ferns and Lycophytes (eds Ranker TA, Haufler CH), pp. 367-394. Cambridge University Press, Cambridge.

Pang XA, Liu X, Liu H, Wu C, Wang JY, Yang SX, Wang QF (2003) The geographic distribution and habitat of the Isoëtes plants in China. Biodiversity Science, 11, 288-294. (in Chinese with English abstract) [庞新安, 刘星, 刘虹, 吴翠, 王晶苑, 杨书香, 王青锋 (2003) 中国三种水非属植物的 地理分布与生境特征. 生物多样性, 11, 288-294.]

Parris BS, Kiew R, Chung RCK, Saw LG, Soepadmo E (2010) Flora of Peninsular Malaysia. Series 1: Ferns and Lycophytes, Volume 1. Forest Research Institute Malaysia, 
Kepong, Selangor Darul Ehsan.

Pryer KM, Schneider H, Smith AR, Cranfill R, Wolf PG, Hunt JS, Sipes SD (2001) Horsetails and ferns are a monophyletic group and the closest living relatives to seed plants. Nature, 409, 618-622.

Smith AR, Pryer KM, Schuettpelz E, Korall P, Schneider H, Wolf PG (2006) A classification for extant ferns. Taxon, 55, 705-731.

Song ZS, Xu RZ, Zhang QH (1989) Rare, Endangered, and Protected Plants in China. China Forestry Publishing House, Beijing. (in Chinese) [宋朝枢, 徐荣章, 张清华 (1989) 中 国珍稀濒危保护植物. 中国林业出版社, 北京.]

Tryon RM, Tryon AF (1982) Ferns and Allied Plants with Special Reference to Tropical America. Springer-Verlag, New York.

Wang JC, Chiou WL, Chang HM (2012) A Preliminary Red List of Taiwanese Vascular Plants. Endemic Species Research Institute \& Taiwan Society of Plant Systematics, Nantou, Taiwan.

Wu SH, Ching RC (1991) Fern Families and Genera of China. Science Press, Beijing. (in Chinese) [ 吴兆洪, 秦仁昌 (1991) 中国硕类植物科属志. 科学出版社, 北京.]

Wu ZY, Raven PH, Hong DY (2013) Flora of China, Vol. 2-3. Science Press, Beijing; Missouri Botanical Garden Press, St. Louis.

Yan YH, Zhang XC, Ma KP (2013) Pteridophytes in China: Diversity and Distribution. Science Press, Beijing. [严岳鸿, 张宪春, 马克平 (2013) 中国烣类植物多样性与地理分 布. 科学出版社, 北京.]

Yan YH, Zhang XC, Zhou XL, Sun JQ (2016) Species Catalogue of China, Vol. 1. Plant: Pteridophytes. Science Press, Beijing. (in Chinese) [严岳鸿, 张宪春, 周喜乐, 孙久琼 (2016) 中国生物物种名录 (第一卷) 植物 烣类植物. 科
学出版社, 北京.]

Yu LF, Gao J, He R, Du JJ, You JY, Deng DZ (2015) Discussion on investigations of the present status of Cystoathyrium chinensis resources in Tianquan and protection measures. Journal of Sichuan Forestry Science and Technology, 36(3), 120-125. (in Chinese with English abstract) [余凌帆, 高健, 何让, 杜俊杰, 尤继勇, 邓东周 (2015) 天全县光 叶偋资源现状调查与保护对策研讨. 四川林业科技, 36(3), 120-125.]

Yu YF (1999) A milestone of wild plants protection in China - The Catalogue of National Key Protected Wild Plants (Part I). Plants, (5), 3-11. (in Chinese) [于永福 (1999) 中 国野生植物保护工作的里程碑一《国家重点保护野生 植物名录(第一批)》出台. 植物杂志, (5), 3-11.]

Zhang XC (2012) Lycophytes and Ferns of China. Peking University Press, Beijing. (in Chinese) [张宪春 (2012) 中 国石松类和硕类植物. 北京大学出版社, 北京.]

Zhang LB, Taylor WC (2013) Isoëtaceae. In: Flora of China, Vol. 2-3 (eds Wu ZY, Raven PH, Hong DY), pp. 35-36. Science Press, Beijing; Missouri Botanical Garden Press, St. Louis.

Zhang XC (2013) Ferns. In: Rare and Endangered Plants in China (ed. Yin H), pp. 6-41. China Forestry Publishing House, Beijing. (in Chinese) [张宪春 (2013) 蕨类植物. 见: 中国珍稀濒危植物图鉴(印红 主编), 6-41页. 中国林 业出版社, 北京.]

Zhang XC, Mickel JT (2013) Schizaeaceae. In: Flora of China, Vol. 2-3 (eds Wu ZY, Raven PH, Hong DY), p. 122. Science Press, Beijing; Missouri Botanical Garden Press, St. Louis. 